\title{
Generating and Supporting Dynamic Heterogeneous MAS
}

\author{
Glenn Tesla Jayaputera, Arkady Zaslavsky, and Seng Wai Loke
}

\begin{abstract}
One of the grand challenges in agent technology research is quasi-automatic development of heterogeneous and dynamic multiagent systems using Agent Oriented Software Engineering (AOSE) tools. This paper presents an innovative approach to developing and supporting multiagent systems at run-time. Multiagent systems (MASs) can and should be generated dynamically based on high-level user specifications which are transformed into a mission. Dynamically generating agents could also be offered as a pervasive service. Heterogeneity of MASs refers to diverse functionality and constituency of the system which include mobile as well as host associated software agents. This paper proposes and demonstrates on-demand and just-in-time agent generation approach which is combined with run-time support for MASs. Run-time support is based on mission cost-efficiency and objectives which enable termination, generation, injection and replacement of software agents as the mission evolves. We present the formal underpinning of our approach and describe the prototype tool - called eHermes, which has been implemented using specific agent platforms. Lessons and results of testing eHermes are reported and analyzed.
\end{abstract}

Index Terms-Mission, On-Demand, Run-Time Support.

\section{INTRODUCTION}

$\mathrm{S}$ ince the early introduction of MASs, a number of systems have been developed and proposed. In general, MASs are developed for specific application domains, and traditionally such systems have a fixed number of agents with fixed functionalities. While such systems are adequate for their purposes, they are not flexible enough to adapt to the changes in their environments. As a result, when the environment and/or problem domain is changed or shifted then the systems become inadequate, failures occur and the whole system must be stopped.

One possible approach to address this inflexibility is to have intelligent, adaptive and relatively complex agents in the systems, where such agents adapt themselves to the changes in their environments or take up new functionalities to work in

Manuscript received April 24, 2006. This work was supported in part by the U.S. Department of Commerce under Grant BS123456 (sponsor and financial support acknowledgment goes here).

Glenn Tesla Jayaputera and Arkady Zaslavsky are with the CSIT, Monash University, 900 Dandenong Road, Caulfield East, Victoria 3145, Australia (email: \{glenn.jayaputera, arkady.zaslavsky\}@infotech.monash.edu.au).

Seng Wai Loke is with the Department of Computer Science and Computer Engineering, La Trobe University, Bundoora, Victoria 3086, Australia (email: s.loke@ latrobe.edu.au). the new problem domains. Another approach is to generate a set of relatively simple but specialized agents on-demand and at run-time dynamically from a given precise plan. Hence, depending on the problem domain, different types of agents are constructed. These agents are given functionalities that are suitable for the problem at hand. In addition, if the existing agents are not adequate anymore (given that they only have specialized functionality) - e.g. due to changes in the environment - then a new set of (specialized) agents can be constructed dynamically to replace them. Hence, agents in this approach are created when they are required and removed from the system when they cannot be utilized anymore. We refer this concept to as on-demand and just-in-time agent generation. Furthermore, the plan must be modifiable at runtime and run-time support infrastructure must be provided so that the system execution can be suspended and resumed later. We explore this approach in this paper.

Our research explores, proposes, develops and analyzes a technique for building MASs which have the following features: (i) given a request, a mission which contains a plan is generated, (ii) a set of relatively simple agents but with specialized functionality is generated from the current plan, (iii) inadequate agents can be replaced at run-time, (iv) the current plan can be modified at run-time, (v) execution can be suspended and resumed, (vi) missions can be migrated to different locations, and (vii) the execution history is maintained (and may be used by the planning mechanism for improvements in the future).

The rest of this paper is organized as follows. Section 2 illustrates the conceptual overview. Section 3 presents the formal model of the mission. Section 4 presents eHermes, the prototype system. Section 5 shows some of the experimental results. Section 6 presents related work. Finally, Section 7 concludes this paper with future work.

\section{CONCEPTUAL Overview}

The conceptual view of our proposed system is depicted in Fig. 1. A mission is created when there is a request. From the request, an initial plan is created by a planner and the plan is regarded as the best plan that can be generated at that time. The mission is created from the initial plan. A mission consists of a current plan, a set of agents working for the mission and other elements such as Mission Data Space and Mission Execution History (formally defined later). For each mission, 
there is a special agent called MCA (Mission Control Agent) which manages and controls the mission execution.

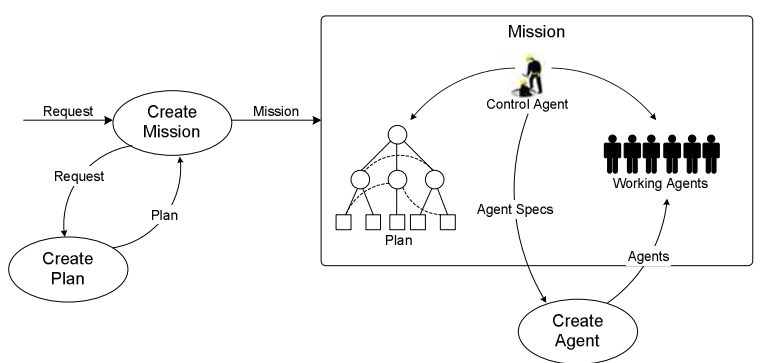

Fig. 1. eHermes System Conceptual Overview.

Although the initial plan is the best plan, in general it can fail and/or be incomplete. Hence, during the mission execution the MCA has the responsibility to modify the plan by adding and/or removing tasks or sub-plans. MCA does not execute the mission by itself, but with the help of a group of agents that are created dynamically for the mission. The agent creation process is initiated by the MCA. In our approach, both stationary and mobile agents are used. The MCA also manages all the working agents' activities such as synchronizing the tasks execution so that there is always enough agents to carry out the mission, allocating tasks to the agents and orchestrating the activities to suspend/resume the mission. The MCA however, does not control how the agents carry out the task(s) given. All the working agents are autonomous agents that can move to different locations if the tasks they are assigned have to be executed at those locations.

\section{MisSION Formal MODEL}

Traditionally, agents are constructed at design phase where their functionality and capability are defined. Generally, these agents are instantiated when the system is executed. Furthermore, in general, their functionality and capability are fixed.

In contrast to such a traditional approach, our approach emphasizes the concept where agents are created when there is a mission. The agents are created when they are required to perform some task(s). These agents terminate themselves once they have completed the given tasks. Hence, our approach guarantees that no agent exists in the system when there is no task to carry out. Such an approach reduces system resource consumption.

A mission must have a goal and a plan to achieve that goal. The plan can be complete or partial. For a system to be able to understand a plan unambiguously, the plan must be represented in a structured and concise form. A plan is regarded as a set of inter-related tasks that must be performed to achieve the objective/goal of the mission. The purpose of capturing the interrelationships between tasks is to specify which tasks must be performed first before others. The purpose of capturing the task decomposition is not only to define how complex tasks split into simpler ones but also to retain the deliberation on the decomposition itself. A task is formally defined as follows:

\section{DEFINITION 1: Task Definition}

Let $N$ be a set of locations at which the tasks must be carried out,

$Y$ be a set of task types, i.e. $Y=\{$ Primitive, Compound\},

$O$ be a set of functions (which represent the application logic that a task will execute),

$S$ be a set of task status, i.e. $S=\{$ Completed, Pending, InProgress, Failed, Aborted, Assigned\}, and,

$U$ is a set of unique IDs in string form.

Then, a task is defined as a tuple of the form $(u, N, y, s, o)$, where $u \in U, y \in Y, s \in S, o \in O$.

There are two types of tasks in our model: compound and primitive. A primitive task is the lowest level of task. A primitive task cannot be broken down further and is directly executed by the system. A compound task, on the other hand, is regarded as a complex task that may or may not contain operation constructs and can be decomposed further into one or more compound and/or primitive task(s). The status of a task is an indicative of its condition at any point in time and it can be any of the following:

- Completed indicates that the task has been completed successfully.

- Pending indicates that the task is ready for execution, and is waiting to be assigned to an agent.

- InProgress indicates that the task is currently being executed.

- Failed indicates that the task has failed when executed.

- Aborted indicates that the task has been aborted and its existence in the mission will be ignored.

- Assigned indicates that the task has been assigned to an agent but has not been carried out yet.

Fig. 2 shows the transition diagram of the task status. At the beginning, the status of a task is Pending and changed to Assigned once it has been allocated to an agent. However, if it is cancelled for any reason then its status becomes Aborted. A task with status Assigned, Pending and InProgress can be aborted at any time. Once a task is executed, its status is changed to InProgress. From this point, if it does not get aborted then its status becomes either Completed or Failed.

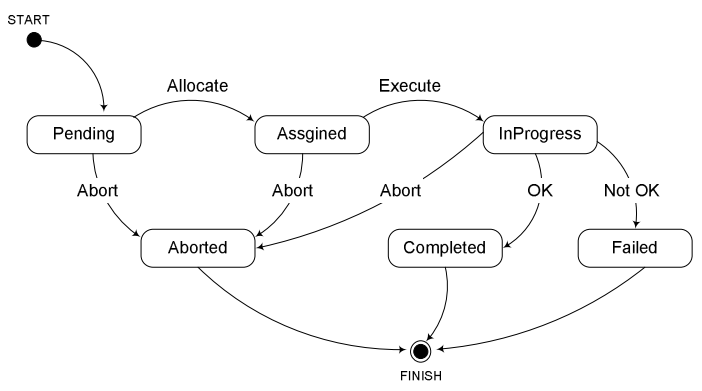

Fig. 2. Task's Status Transition Diagram illustrates the life-cycle of the tasks.

Tasks are interrelated to each other and these relationships are captured by the links that connect them. Hence, a link 
between two tasks represents the relationship that exists between them. A link is defined as follows:

\section{DEFINITION 2: Link Definition}

Let $Q$ be the type of the relationship, $Q=\{$ Includes, DependsOn\}, and

$T$ be a set of tasks as defined in Definition 1,

Then a link is defined as a tuple of the form $\left(t_{i}, t_{j}, q\right)$

Where $i, j \in \mathbb{Z}^{+}, q \in Q, i \neq j, t_{i}, t_{j} \in T$.

There are two types of links: Includes and DependsOn. The DependsOn link specifies the dependency between two tasks the link connects. When a task depends on another then that task cannot be executed until the task it depends on has been executed successfully. Therefore, a DependsOn relationship between tasks A and B (A DependsOn B) means that task B must be completed first (i.e. the status of $\mathrm{B}$ is Completed) before task A can be executed. The Includes link illustrates the inclusiveness of a parent task over its sub-tasks. Hence, an Includes relationship between tasks A and B (A Includes B) means that (i) task B is part of task A and (ii) task A depends on task B.

As indicated earlier, a plan must be represented in a nonambiguous form to avoid any misinterpretation. Furthermore, for our research purposes, the plan must be simple and dynamic so that it can be updated and modified easily at runtime. By allowing plan modification at run-time, plan execution does not have to be terminated prematurely when failures occur. Normally, a plan fails because of either: (i) The assumptions that were made during the planning process were wrong or, (ii) The run-time condition/environment has changed or different from the one that is anticipated at planning time. In any case, it is useful not to abort a mission too soon once it has failed. Instead, it is better to halt the execution, modify the plan and re-execute the mission from the point where it stopped. A plan is defined as follows:

\section{DEFINITION 3: Plan Definition}

A plan is represented in a Directed Acyclic Graph structure called Task Decomposition Graph (TDG). Given $f_{\text {type }}: T \rightarrow Y$, a function that returns the type of a given task then, a TDG is a pair $(T, L)$, where $T$ is a nonempty set of tasks as specified in Definition 1 , and $L$ is a non-empty set of links as specified in Definition 2 such that the following properties are satisfied:

(i) $\forall\left(t_{i}, t_{j}, q\right) \in L, f_{\text {tvpe }}\left(t_{i}\right) \neq$ Primitive

(ii) $\forall\left(t_{i}, t_{j}, q\right) \in L$, if $q=$ Includes then $f_{\text {type }}\left(t_{i}\right)=$ Compound

(iii) $\forall\left(t_{i}, t_{j}, q\right) \in L$, if $f_{\text {type }}\left(t_{j}\right)=$ Compound then $\exists\left(t_{j}, t_{k}, q^{\prime}\right) \in L$ where $t_{i}, t_{j}, t_{k} \in T, q, q^{\prime} \in Q$ and $i, j, k \in \mathbb{Z}^{+}$

The first property specifies that the source of a link cannot be a primitive task. Hence, this implies that a primitive task cannot be decomposed further nor it can depend on any other tasks. The second property specifies that when the type of a link is Includes, the source of that link must be a compound task and the destination can be a compound and/or primitive task. The third property specifies that a compound task cannot be the leaf node in the TDG. A mission is formally specified as follows:

\section{DEFINITION 4: Mission Definition}

A mission is a tuple of the form $(o, P, A, Z)$, where $o$ is the objective of the mission, $P$ is a set plans/TDGs, $A$ is a set of agents working in the mission and $Z$ is a set of mission states (formally defined in Definition 14).

Given $f_{\text {status }}: T \rightarrow S$, a function that return the status of a given task and a mission $m=(o, p, A, Z)$, then a mission is said to be accomplished if the following function returns true.

\section{DEFINITION 5: isAccomplished Function Definition}

$$
f_{\text {istccomplissed }}(m) \Leftarrow \begin{cases}\text { True } & \text { if } \forall t \in T \text { s.t. } f_{\text {status }}(t)=\text { Completed } \\ \text { False } & \text { otherwise }\end{cases}
$$

where $T$ is a set of tasks of plan $p$.

When a mission is executed, it is actually the mission's plan (i.e. the TDG) that is carried out by a set of dynamically created agents. These agents can be either stationary or mobile agents depending on the location at which the task must be carried out. Each of these agents is given a task(s) to complete. The tasks are not executed arbitrarily, but in such a way where the task dependencies and decompositions are honored.

The TDG is not a static structure but dynamic and evolving as the mission is executed. To formally specify all the operations on the TDG the following notations are used:

\section{DEFINITION 6: Plan Evolution Definition}

Let $P=\left\{p_{0}, p_{1}, \ldots, p_{m}\right\}, \mathrm{m} \in \mathbb{Z}^{+}$be the finite set of plans where $p_{0}$ is the initial plan and $p_{m}$ is the final plan. Also, let $F=\left\{f x_{0}, f x_{1}, \ldots, f x_{n}\right\}, n \in \mathbb{Z}^{+}$be the finite set of operations that can be applied to $P$; then, the plan evolution $P E$ is a sequence of interleaved plans and operations such that:

$$
P E: p_{0} \stackrel{f x_{0}}{\longrightarrow} p_{1} \stackrel{f x_{1}}{\longrightarrow} p_{2} \stackrel{f x_{2}}{\longrightarrow} \ldots \stackrel{f x_{n}}{\longrightarrow} p_{m}
$$

Given a plan $p=(T, L)$, the operations that are applicable to $P$ are specified as follows:

(i) Given a task $t=(u, N, y, s, o)$ and a new task status $s^{\prime}$ then $f_{\text {sestatus }}\left(p, t, s^{\prime}\right)$ is an operation that modifies the status of task $t$ from $s$ to $s^{\prime}$ according to the transition rule: $(T, L) \rightarrow\left(T \backslash\{t\} \cup\left\{t_{x}\right\}, L \backslash \delta_{L}^{-} \cup \delta_{L}^{+}\right)$where:

$t_{x}=\left(u, N, y, s^{\prime}, o\right), \delta_{L}^{-}=\left\{\left(t_{i}, t_{j}, q\right) \in L \mid t_{i}=t \vee t_{j}=t\right\}$,

$\delta_{L}^{+}=\left\{\left(t_{x}, t_{j}, q\right) \mid\left(t, t_{j}, q\right) \in \delta_{L}^{-}\right\} \cup\left\{\left(t_{i}, t_{x}, q\right) \mid\left(t_{i}, t, q\right) \in \delta_{L}^{-}\right\}$ and $u \in U, y \in Y, o \in O, s, s^{\prime} \in S, i, j, x \in \mathbb{Z}^{+}$

(ii) $f_{\text {prunePlan }}(p)$ is an operation that cleans the TDG by removing all the completed and aborted tasks according to the transition rule $(T, L) \rightarrow\left(T \backslash T_{\delta}, L \backslash L_{\delta}\right)$ where: 
$T_{\delta}=\left\{t \in T \mid f_{\text {status }}(t)=\right.$ Completed $\vee f_{\text {status }}(t)=$ Aborted $\}$,

$L_{\delta}=\left\{\left(t_{i}, t_{j}, q\right) \in L \mid t_{i} \in T_{\delta} \vee t_{j} \in T_{\delta}\right\}, T_{\delta} \neq \varnothing \wedge L_{\delta} \neq \varnothing$

and $i, j \in \mathbb{Z}^{+}, q \in Q$

(iii) Given a compound task $t=(u, N$, Compound, $s, o)$, $f_{\text {mutateTask }}(p, t)$ is an operation that changes a task of type compound to primitive according to the following transition rule

$$
\begin{gathered}
L_{c}=\left\{\left(t_{i}, t_{j}, q\right) \in L \mid t_{i}=t\right\} \\
\frac{\forall\left(t_{i}, t_{j}, q\right) \in L_{c} \text { s.t. } f_{\text {status }}\left(t_{j}\right)=\text { Completed }}{(T, L) \rightarrow f_{\text {prunePlan }}((T, L)) \rightarrow\left(T \backslash\{t\} \cup\left\{t_{x}\right\}, L \backslash \delta_{L}^{-} \cup \delta_{L}^{+}\right)}
\end{gathered}
$$

where:

$$
\begin{aligned}
& t_{x}=(u, N, \text { Primitive, } s, o), \\
& \delta_{L}^{-}=\left\{\left(t_{i}, t_{j}, q\right) \in L \mid t_{i}=t \vee t_{j}=t\right\}, \\
& \delta_{L}^{+}=\left\{\left(t_{x}, t_{j}, q\right) \mid\left(t, t_{j}, q\right) \in \delta_{L}^{-}\right\} \cup\left\{\left(t_{i}, t_{x}, q\right) \mid\left(t_{i}, t, q\right) \in \delta_{L}^{-}\right\}, \\
& u \in U, o \in O, s \in S, i, j, x \in \mathbb{Z}^{+}, q \in Q
\end{aligned}
$$

The $f_{\text {mutateTask }}()$ operation is pre-empted with the $f_{\text {prunePlan }}()$ operation so that all outgoing links from task $t$ do not need to be replaced and then removed at a later stage (as would be the case if the $f_{\text {pruneplan }}()$ operation is performed after the transition). The algorithm of the $f_{\text {mutateTask }}($ ) operation is shown in Fig. 3. Line 6 of the algorithm gets all the outgoing links from task $t$, while line 8 gets only the outgoing links that are connected to the completed tasks. If the number of links of these two groups is the same then it indicates that all sub-tasks of task $t$ have been completed, and hence $t$ can be mutated. Next, all the completed tasks are removed from the TDG, including all sub-tasks of task $t$. A new primitive task $\left(t_{x}\right)-$ which has all task $t$ 's properties but its type, is created. Then, a list of links that are to be removed from the plan is constructed. This is followed by creating a list of links that are to be added to the plan. Finally, the plan is modified by removing $t$ and its associated links, and adding $t_{x}$ and its associated links.

When selecting a task(s) to be carried out, the TDG is traversed. Since the size of the TDG can be potentially large, it is important to have an efficient mechanism so that the traversal can be carried out as efficiently as possible. To address this challenge, a plan is executed with the following strategies:

- Only execute primitive tasks. Initially the primitive tasks are leaf nodes due to the plan properties specified previously. Given this initial condition, the primitive tasks are easily collected with a simple linear sweeping method.

- Convert a compound task into primitive task when possible. In order to execute the primitive tasks only, compound tasks must be converted into primitive tasks when all their immediate sub-tasks have been completed as presented by the transition rule previously.

- Perform plan trimming operation. As the mission is executed, the tasks becomes completed or aborted. It is necessary to remove those tasks from the plan so that the linear sweeping method can work more efficiently. The logic for pruning the plan has been formally specified previously. Fig. 4 illustrates how the pruning is carried out. Fig. 4(i) show the TDG before pruned. It is assumed that task $\mathrm{B}$ is aborted and tasks $\mathrm{N}, \mathrm{P}$ and $\mathrm{Q}$ have been completed (they are marked with red crosses). Fig. 4(ii) shows the result after the pruning - task $G$ has been converted into a primitive task, and hence is ready to be executed in the next execution cycle.

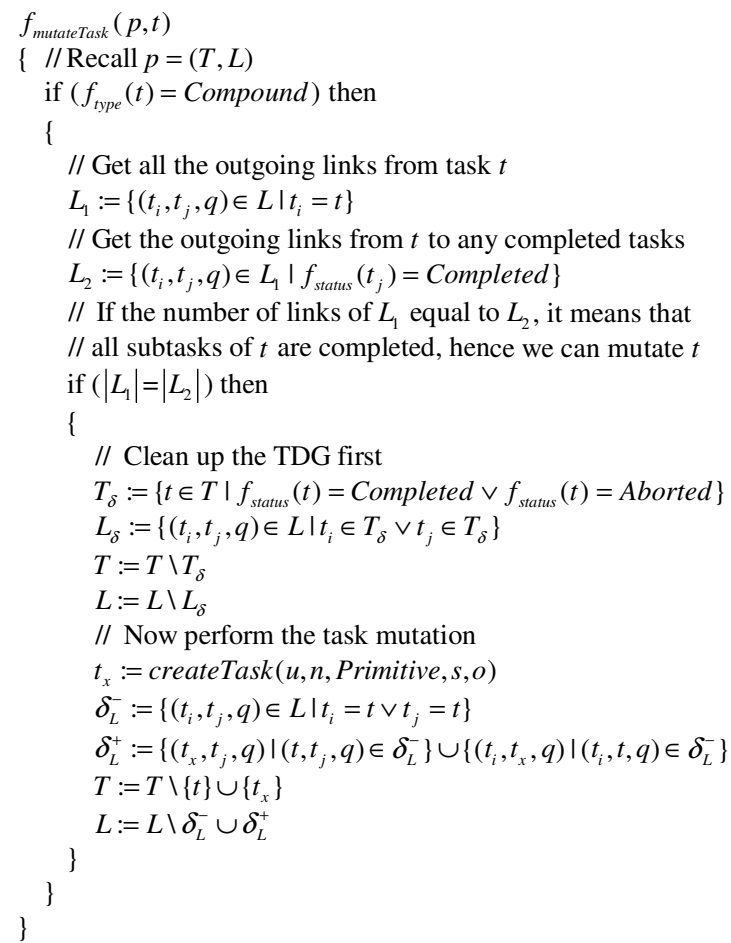

Fig. 3. Task Mutation Algorithm.
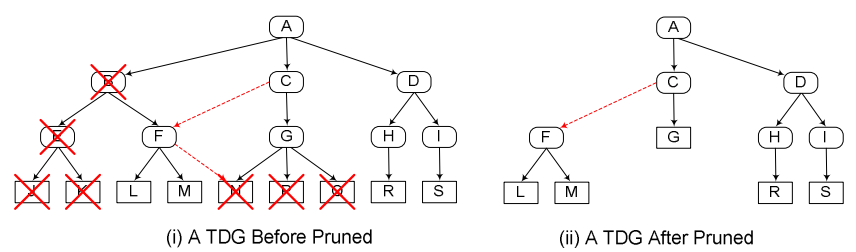

(ii) A TDG After Pruned

Fig. 4. TDG Pruning Illustration.

During the mission execution, a task(s) with either Pending, InProgress or Assigned status can be aborted at any time. When a task is aborted, the following actions will take place:

- If the task(s) have been allocated to an agent or are being executed, then the relevant agent will be informed. The agent will either abort the tasks (if it is being executed) or remove the task(s) from its "to do" list.

- The tasks and their relevant sub-tasks will be tagged with Aborted status and will be pruned from the plan at a later stage.

Special attention must be taken when aborting a task (and all its relevant sub-tasks) so that the task dependencies are not broken and the resulting TDG still conforms to the TDG specification. Therefore, the following rules are put in place: 
(i) a task can be aborted at any time, even when it is being executed, (ii) a task cannot be aborted if there is a task(s) that depend on it, and finally (iii) A task can only be tagged as Aborted if and only if all of its sub-tasks have been aborted. To elaborate the operation, consider the simple TDG structure depicted in Fig. 5.

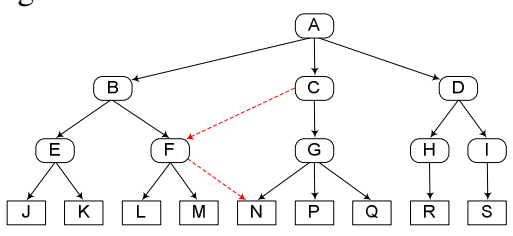

Fig. 5. Run-Time Task Abortion.

The simplest scenario is to abort task P. This task can be aborted without any concern. The situation will be different if the task is N. Since there is a task (i.e. task F) that depends on this task, then this task cannot be freely aborted - otherwise the mission execution cannot be completed. In this situation, task $\mathrm{N}$ cannot be aborted and is let to continue. When a compound task is aborted, the system will abort all the tasks that are directly or not directly decomposed from that task. For instance, if task D is aborted than the sub-graph whose root node is this task, is aborted. That is, tasks D, H, I, R, and S are aborted. The situation can be more complex as in the case where task B is aborted. In this case, the whole sub-graph can not be aborted because task $\mathrm{C}$ depends on task $\mathrm{F}$ and task $\mathrm{F}$ cannot be completed until task L, M and $\mathrm{N}$ are completed. Hence, the only tasks that are aborted in this case are tasks B, $\mathrm{E}, \mathrm{J}$ and $\mathrm{K}$. The algorithm to abort a task is shown in Fig. 6.

$$
\begin{aligned}
& f_{\text {abort }}\left(p, t_{i}\right) \\
& \{ \\
& \text { DLSet }:=\left\{\left(t_{j}, t_{k}, q\right) \in L \mid t_{k}=t_{i} \wedge q=\text { DependsOn }\right\} ; \\
& \text { if }(D L S e t \neq \varnothing) \\
& \text {; //Stop traversing and do nothing } \\
& \text { else } \\
& \{ \\
& \text { ILSet }:=\left\{\left(t_{l}, t_{m}, q\right) \in L \mid t_{l}=t_{i} \wedge q=\text { Includes }\right\} ; \\
& \forall\left(t_{l}, t_{m}, q\right) \in I L S e t \text { do } \\
& f_{\text {abort }}\left(t_{m}\right) \text {; } \\
& f_{\text {setStatus }}\left(p, t_{i}, T S_{-} A B O R T E D\right) \text {; } \\
& \text { \} }
\end{aligned}
$$$$
\}
$$

Fig. 6. Task Abortion Algorithm.

As indicated before, a mission is executed by performing the plan specified in that mission. The plan is executed by strata and a stratum is defined as follows:

\section{DEFINITION 7: Stratum Definition}

Given $p=(T, L)$, the current plan of a mission, $a$ stratum(st) is defined as a set of primitive tasks whose statuses are Pending, and hence:

$$
s t=\left\{t \in T \mid f_{\text {type }}(t)=\text { Primitive } \wedge f_{\text {status }}(t)=\text { Pending }\right\}
$$

The concept of the stratum is not the same as the concept of level established in graph theory. A level in the graph theory is used to specify the depth of a node from the root node [1]. In our approach, we assume that for every single agent that exists in the system, there is a cost involved. The metric used to calculate the cost in this context is the amount of system resources consumed. This includes the resources required to create an agent(s), move the agent(s) to the preferred location(s) and to have communication between the agents. Hence, in order to carry out a mission efficiently the right number of agents is required. To have more than the necessary number of agents does not generally guarantee that the mission will be completed sooner or better. This situation is analogous to a simple fact that to have one hundred plumbers to fix a leakage in a kitchen sink does not necessary mean that the job can be done faster - because there is only a limited space underneath the sink so that it is practically impossible to fit all the plumbers underneath the sink. On the other hand, to extremely minimize the number of agents so that only one agent performs the whole mission might mean parallelism is not fully exploited, thereby delaying mission completion. Our approach to have the right number of agents working in a mission is in line with our on-demand agent generation concept.

In order to address the challenge of having the right number of agent working in the mission with as little impact as possible to the mission completion time, we use the Cost/Benefit Analysis [2] concept in order to determine the future course of action. The Cost/Benefit Analysis (CBA) concept is relatively simple yet widely used in determining whether the change we are going to introduce is a worthwhile effort. The CBA concept simply tries to reduce the cost level, and at the same time tries to maximize or maintain the current benefit level. The notion of the cost and benefit differs from one problem domain to another. In our context, the "cost" is associated with the number of agents created for a given mission. Hence, for our purposes, the number of agents generated needs to be minimized as much as possible. Subsequently, the "benefit" is the mission completion elapsed time. Hence, in order to get the maximum benefit, we must minimize the delays that might occur as the number of agents is reduced.

Recall that tasks are executed by strata, and hence, given strata $S T=\left\{s t_{1}, s t_{2}, \ldots, s t_{n}\right\}$ where $n \in \mathbb{Z}^{+}$then $s t_{n}$ will not be executed until $s t_{n-1}$ is completed. For a stratum to be classified as completed, all of the tasks contained must be completed. It is assumed that all the tasks in a plan are designed for different purpose(s) - hence, they have different elapsed times. The task that has the biggest elapsed time in a stratum is considered to be the critical task of that stratum. Hence, even though all other tasks in that stratum have been completed, the next stratum cannot be computed until the critical task(s) are completed. The definition of the task cluster is presented as follows:

\section{DEFINITION 8: Task Clusters for a Stratum Definition}

Given a stratum st where $s t \in S T$, a collection of task clusters is defined as a collection of disjoint subsets of $s t$, 
$X_{1}, X_{2}, \ldots, X_{n}$ such that $X_{i} \subseteq s t, s t=\bigcup_{i=1}^{n} X_{i}, X_{i} \cap X_{j}=\varnothing$

where $i, j, n \in \mathbb{Z}^{+}, i \neq j$.

The cost to execute a task cluster and a mission are defined as follows:

\section{DEFINITION 9: Task Cluster Execution Cost Definition}

Given a task cluster

$X=\left(\left(u_{1}, N_{1}, y_{1}, s_{1}, o_{1}\right),\left(u_{2}, N_{2}, y_{2}, s_{2}, o_{2}\right), \ldots\left(u_{k}, N_{k}, y_{k}, s_{k}, o_{k}\right)\right\}$

where $k \in \mathbb{Z}^{+}$, let $N x$ be a set of locations at which the tasks in $X$ must be executed, i.e. $N_{x}=N_{1} \cup N_{2} \cup \ldots N_{k}$. The cost of executing the task cluster is then defined as the sum of the cost to create an agent, the total cost to execute all the tasks in the task cluster, the total cost to move to the locations at which the tasks must be executed and the cost of data transfer (such as the cost for sending the results back to the system and the communication cost between the agents). Hence, the cost is given by:

$$
\begin{gathered}
f_{\text {cost }}(X)=f_{\text {genAgent }}(X)+\sum_{i=1}^{|X|} f_{\text {execute }}\left(\left(u_{i}, N_{i}, y_{i}, s_{i}, o_{i}\right)\right)+ \\
\sum_{i=1}^{|N x|} f_{\text {move }}\left(N_{i}\right)+\sum_{i=1}^{|X|} f_{\text {dataXfer }}\left(c_{i}+r_{i}\right)
\end{gathered}
$$

where

$c_{i}$ is the communication cost related to task $\left(u_{i}, N_{i}, y_{i}, s_{i}, o_{i}\right), \quad r_{i}$ is the result(s) produced by task $\left(u_{i}, N_{i}, y_{i}, s_{i}, o_{i}\right)$ and $u \in U, y \in Y, s \in S, o \in O, i \in \mathbb{Z}^{+}$.

\section{DEFINITION 10: Mission Execution Cost Definition}

Given a mission with a plan $p=(T, L)$ and a collection of task clusters $X_{1}, X_{2}, \ldots, X_{k}, k \in \mathbb{Z}^{+}$, the cost to execute the mission is the total cost to execute all those task clusters. Hence,

$$
f_{\text {cost }}(p)=\sum_{i=1}^{k} f_{\text {cost }}\left(X_{i}\right)
$$

We assume at the present moment that the mission is executed within a high speed local network infrastructure. Hence, the cost of data transfer which is contributed by the agent's mobility, sending and receiving results and agent communication, can be ignored. This simplification allows us to focus on the trade-off between cost due to generating agents and parallelism. Recall that to execute a mission efficiently, each agent a task cluster as opposed to a single task. Hence, minimizing the number of agents needed means minimizing the number of task clusters created. Hence, the following definitions are presented.

\section{DEFINITION 11: Stratum Critical Time Definition}

Given $f_{\text {elapsed }}: T \rightarrow \mathbb{Z}^{+}$, a function that returns the elapsed time of a given task (in some time units), the critical time of a stratum st (denoted by $C T_{s t}$ ) where $s t \in S T$ is defined as the maximum elapsed time of all the tasks in st. Hence:

$$
C T_{s t}=\max \left\{\forall t \in s t, f_{\text {elapsed }}(t)\right\}
$$

\section{DEFINITION 12: Mission Critical Time Definition}

Given a plan $p=(T, L)$ and strata $S T=\left\{s t_{1}, s t_{2}, \ldots, s t_{k}\right\}$ where $k \in \mathbb{Z}^{+}$, the mission critical time $C T_{m}$ is defined as the sum of the critical time of the strata, hence:

$$
C T_{m}=\sum_{i=1}^{k} C T_{s t_{i}}
$$

Grouping tasks in a stratum st such that the critical time of each group does not exceed $C T_{s t}$ is considered as an off-line Bin-Packing Problem (BPP). Finding an optimal algorithm to address such a challenge is NP-hard. However, there are a number of near optimal algorithms available. In general, FirstFit Decreasing (FFD) [3] is considered to be the paramount among all of them. Using this approach, tasks in each stratum is sorted in decreasing order according to their elapsed times. Therefore, the task that takes the longest time to complete will be the first in the sorted list. The critical time of the stratum

\begin{tabular}{|c|c|}
\hline 1 & $f_{\text {costBenefitExecute }}(p)$ \\
\hline 2 & \{ \\
\hline 3 & $/ /$ Recall that $p=(T, L)$ \\
\hline 4 & while $\left(T \neq \varnothing \wedge f_{\text {isAborted }}()=\right.$ false $)$ \\
\hline 5 & \{ \\
\hline 6 & $s t:=\left\{t \in T \mid f_{\text {type }}(t)=\right.$ Primitive $\wedge f_{\text {status }}(t)=$ Pending $\}$ \\
\hline 7 & $s t_{\text {sorted }}:=f_{\text {sort }}\left(f_{\text {quicksort }}, f_{\text {elapsed }}, s t\right)$ \\
\hline 8 & $X:=f_{F F D}\left(s t_{\text {sorted }}\right)$ \\
\hline 9 & $\forall x \in X$ do \\
\hline 10 & \{ \\
\hline 11 & $a:=f_{\text {createAgent }}(x)$ \\
\hline 12 & $f_{\text {sendCommand }}(a$, CMD_EXECUTE) \\
\hline 13 & \} \\
\hline 14 & $f_{\text {sleep }}\left(f_{\text {elapsed }}(t)\right) \quad / /$ where $t \in x_{1}$ \\
\hline & \} \\
\hline 16 & \}$/ /$ End Of $f_{\cos \text { BenefitExecute }()}$ \\
\hline
\end{tabular}
$\left(C T_{s t}\right)$ is then equal to $f_{\text {elapsed }}\left(t_{1}\right), t_{1} \in s t$. The rest of the tasks in st are then grouped into clusters such that the critical time of each cluster is less than or equal to $f_{\text {elapsed }}\left(t_{1}\right)$. Fig. 7 shows the algorithm to execute the mission cost efficiently.

Fig. 7. CostBenefit Mission Execution Algorithm. This algorithm considers the cost-efficiency when running a mission. The right amount of worker agents will be created when the mission is executed with this algorithm.

Line 6 of the algorithm presented in Fig. 7 creates a stratum called st. Line 7 sorts the tasks of stratum st based on their elapsed time using Quicksort. The result of this operation is placed into a container called $s t_{\text {sorted }}$. A collection of task clusters $(X)$ is created on line 8 using the FDD algorithm. From lines $9-13$, for each of these task clusters, an agent is created. Then, these agents are instructed to carry out the tasks given. This thread of execution is put into a sleep mode until awakened or a time out has occurred. The time out is set to the critical time of the given stratum (line 14). In this prototype, 
we use Quicksort to sort the tasks in each stratum. This is because in general Quicksort runs reasonably quickly. However, this does not imply that Quicksort must be used, in fact further research can be done to find the most suitable combination of the Bin-Packing and sorting algorithms to be used. On average, the time complexity of the cost efficient mission execution algorithm is $O(n \log n)$ and $O\left(n^{2}\right)$ in the worst scenario, for $n$ being the number of tasks. The complexity time is directly related to Quicksort as indicated in $[1,4]$.

When the tasks are executed, they may or may not produce results/output, and these results may or may not be required by other agents. Acknowledging this condition, we design a placeholder for the agents to exchange data, and call this placeholder Mission Data Space (MDS). The idea behind MDS is similar to that of JavaSpace ${ }^{\mathrm{TM}}$ except MDS is local to a mission. The MDS is defined as follows:

\section{Definition 13: Mission Data Space Definition}

A Mission Data Space (MDS) is a set of tuples of the form $\left(u_{t}, r, t_{s}\right)$ where:

$u_{t} \quad$ is the UID of task $t \in T$ and $T$ is a set of tasks,

$r \in R$ and $R$ is a set of results/output, and

$t_{s} \quad$ is the timestamp at which $r$ was produced.

Besides identifying the necessity to have a space where the agents can exchange their data, we also recognize the importance of capturing and preserving the mission execution history. In our concept, preserving the mission execution history means maintaining the state and data of the mission. Hence, the structure of the plan and the state and data of the tasks have to be preserved. Let $E=\left\{e_{1}, e_{2}, \ldots, e_{k}\right\}, k \in \mathbb{Z}^{+}$be the finite set of events that can trigger transitions and $M S=\left\{m s_{1}, m s_{2}, \ldots, m s_{m}\right\}, m \in \mathbb{Z}^{+}$be a set of mission states, then a mission execution $M E$ is a sequence of interleaved mission states and events such that:

$$
M E: m s_{1} \stackrel{e_{1}}{\longrightarrow} m s_{2} \stackrel{e_{2}}{\longrightarrow} \ldots \stackrel{e_{k}}{\longrightarrow} m s_{m}
$$

\section{DEFINITION 14: Mission State Definition}

A mission state $z \in Z$ is a snapshot of the state and data of the mission and is as a tuple of the form ( $\left.p, m d s, e, t_{s}\right)$ where:

$p \quad$ is the current plan as defined in Definition 3, $m d s$ is a snapshot of the MDS at time $t_{s}$,

$e \quad$ is the event that caused the transition, $e \in E$ and $E=\{$ Suspend, Stop, Resume, Start, ChangePlan $\}$,

$t_{s} \quad$ is the timestamp of the transition.

As indicated previously. Plans must be able to be modified at run-time when failures occurred. The run-time plan modification operation is formally specified as follows:

DEFINITION 15: Run-Time Plan Modification Definition Given a plan $p=(T, L)$, let $\Delta$ be a set of tasks and links that are to be added to and removed from $p$. Also, let $\delta_{T}=\{t \mid t \in \Delta\}$ is a set of tasks to be added to and removed from $p$, and $\delta_{L}=\left\{\left(t_{i}, t_{j}, q\right) \mid\left(t_{i}, t_{j}, q\right) \in \Delta\right\}$ is a set of links to be added to and removed from $p$, where $i, j \in \mathbb{Z}^{+}, q \in Q$. Then, a plan modification operation is defined as the following transition rule:

$$
(T, L) \rightarrow\left(\left(T \cup \delta_{T}\right) \backslash\left(T \cap \delta_{T}\right),\left(L \cup \delta_{L}\right) \backslash\left(L \cap \delta_{L}\right)\right)
$$

\section{EHERMES: MISSION-BASED MAS GENERATOR}

eHermes is the prototype system being developed based of the theory and concept discussed. The Grasshopper agent development toolkit [5] is used because it has suitable infrastructures to support mobile agents. The architecture of the eHermes system is presented in Fig. 8.

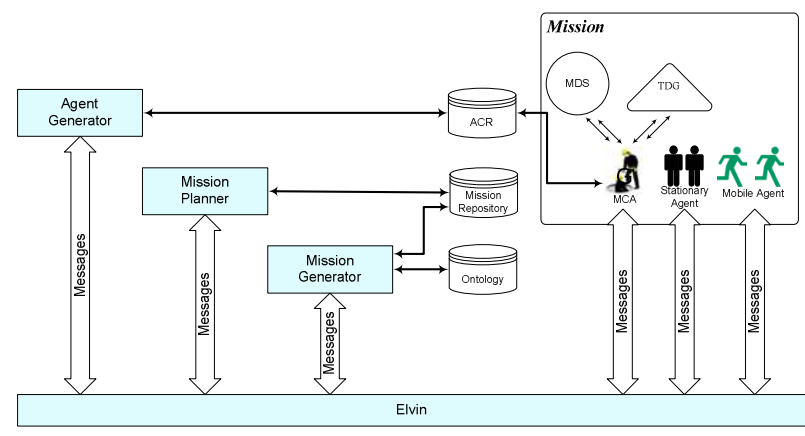

Fig. 8. The eHermes System Architecture.

The eHermes system comprises several major components: Mission Generator, Mission Planner, Agent Generator and Elvin [6]. Mission Generator is the module that is in charge of generating mission objects. A mission object is generated in response to a high level user request. The mission generator uses an ontology in order to interpret the request properly. In addition to that, the mission generator will reuse existing missions if one exists. The mission generator is not equipped with the capability to generate plans - hence it relies on the mission planner to generate an initial plan. The mission planner does not always generate plans from scratch but makes use of an existing plan if available. We assume at this stage that the mission planner can generate a plan in a reasonable time frame. Finding the best planning algorithm is beyond the scope of this project.

Once the plan has been successfully generated, it is returned to the mission generator, which then generates a mission object. Next, this mission object is passed on to another module called Agent Generator. The agent generator is the backbone of our on-demand run-time agent generation system. Its main task is to generate agent(s) upon requests, and hence, this module is not responsible for coordinating or managing the agents it generates. Upon receiving the mission object, the agent generator creates the special agent called MCA. MCA's main responsibility is to coordinate/manage the other agents working for the mission, and provide a point of contact for the user. When composing the agents, the agent generator utilizes a repository that contains a collection of modules called Agent Component Repository (ACR). MCA does not execute the mission by itself, but rather through a number of agents. These 
agents are created dynamically when needed. Furthermore, these agents can be either mobile and/or stationary agents depending on the locations at which the task(s) must be executed. Tasks are executed concurrently in a strata fashion as specified in the previous section.

eHermes agents are not interpreters - hence, the task specifications must be compiled into executable entities so that the agents can execute them. The agent generator produces executable entities from the given task specifications. The task specification is defined by an instance of eHTaskspec class. Two of this class attributes - namely: szLibCodes and szLibName, specify the computation logic of the task. szLibCodes attribute provides a placeholder where programming language code can be stored. szLibName attribute is used to specify which ACR (Agent Component Repository) component(s) this task uses. If these two attributes are specified, then szLibCodes takes precedence over szLibName. szLibCodes attribute can contain Java code segment. If szLibName is used, the agent generator simply loads the module specified by szLibName from the ACR. However, if szLibCodes is used then additional work must be carried out by the agent generator. In this case, the agent generator must produce Java source code for the task so that it can be compiled into an executable entity. The agent generator uses the template shown in Fig. 9 to generate the source code.

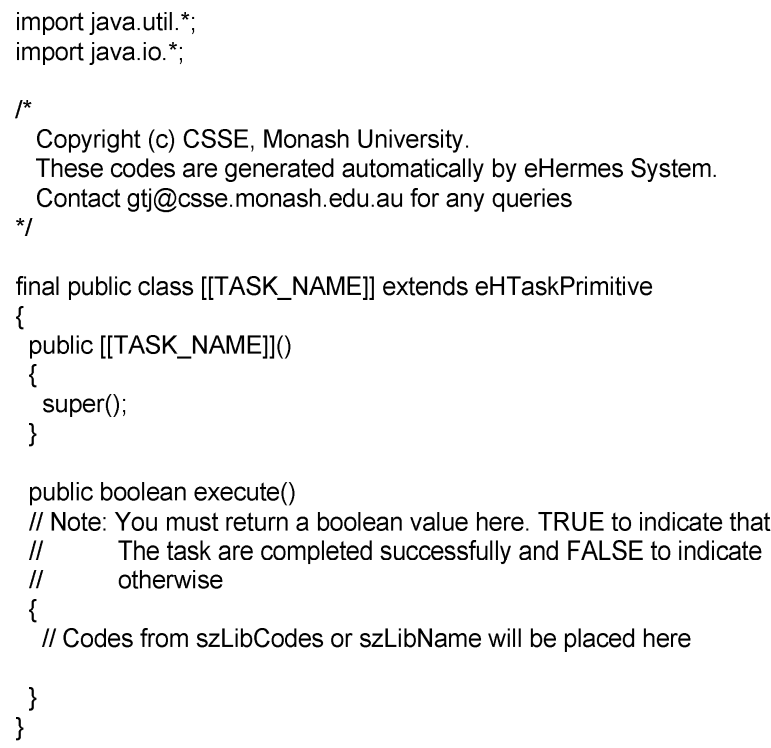

Fig. 9. Task Source Code Template. This template is used by the agent generator to produce source code for a task.

The [ [TASK_NAME]] tag string of this template is substituted with the task's name. The Java code segment retrieved from the szLibCodes attribute is placed inside the execute () method. After the code is generated, it is automatically compiled with a Java compiler.

Once the task is composed successfully, the Agent Generator continues the process by creating an appropriate agent - mobile or stationary agent. Unlike composing the tasks, agents are not produced by generating and compiling code, but rather by simply loading the generic agent class at run-time. Because the eHermes system uses the Grasshopper agent development toolkit, the Grasshopper's mechanism to produce agents is used. The following code fragment shows how agents are created in the eHermes system.

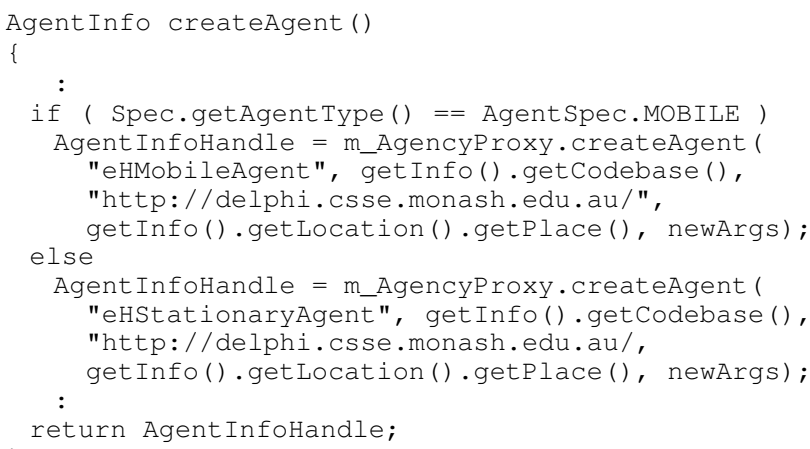

The code fragment above shows that Grasshopper loads an instance class of either eHMobileAgent or eHStationaryAgent - depending of the given agent specification. A mobile agent is composed if the type is AgentSpec.Mobile, otherwise a stationary agent will be composed. The createAgent() method returns a handle (AgentInfoHandle) to an object that contains information about the agent that had just been created. From this information object, the agent UID can be retrieved if necessary. Once created, the agent load any given task(s) into the system using Java class loader mechanism. The following code fragment shows how a task object - which name is specified by the tLibName variable is loaded into the system.

Class TaskClass = Class.forName (tLibName);

m_Task = (eHTaskAbstract) TaskClass.newInstance ();

Tasks are executed by calling their execute () methods. For instance, to execute the m_Task task above, the agent simply calls m_Task.execute(). Tasks are not able to store and retrieve values to and from the MDS directly. A task must request the agent that is executing it to perform such operations. All eHermes agents provide to two methods namely putTaskResult() and getTaskResult(), which can be used to store and retrieve values from the MDS.

Agents communicate via short notifications and messages as opposed to dialogues. This is necessary because eHermes uses mobile agents, and hence it is crucial to have a short and straight forward communication due the unreliable nature of wireless networks. Elvin messaging system is used because it supports: (i) content-based messages, which is important for sending temporary results to the MDS, (ii) publish/subscribe messaging strategy, which is important so that the agents are not required to have permanent TCP/IP connections, and finally (iii) message quenching and hence is fast, reliable and minimize the network bandwidth usage. It should be noted however, that although Elvin is used in our prototype system it does not mean that other content-based and publish/subscribe messaging systems are not usable. 


\section{EMPIRICAL RESULTS}

In this section, we present the results from the experiments that we have conducted. The aim of the experiments is to observe and study our approaches when put under different situations and workloads. In these experiments different types and complexities of plans are used. The simplest plan is composed of only 16 tasks while the most complex plan consists of over 1500 tasks. Throughout this section the term "optimization" is used and it is referring to cost-efficient mission execution strategy.

\section{A. Optimization Test}

The purpose of this experiment is to observe the behavior of the optimization engine which utilizes the Cost/Benefit Analysis across different types of plan structure. Two parameters, $n$ and $m$ are used to adjust the width and height of the TDG. Parameter $n$ is used to specify the number of child nodes that a parent node can have, while parameter $m$ is used to specify the depth of the TDG. Therefore, when $n=1$ the structure of the TDG becomes linear. In this experiment, each agent is assigned with one or more tasks to retrieve information from a medium size database. The results of this experiment are shown in Table 1.

TABLE I

Agent Count REDUCTION TEST REsults

\begin{tabular}{cccccc}
\hline $\begin{array}{c}\text { Test } \\
\text { No }\end{array}$ & $\begin{array}{c}\text { Plan Structure } \\
\text { Parameters }\end{array}$ & $\begin{array}{c}\text { No Of } \\
\text { Tasks }\end{array}$ & $\begin{array}{c}\text { Agent Count } \\
\text { Without Optimization }\end{array}$ & $\begin{array}{c}\text { Agent Count } \\
\text { With Optimization }\end{array}$ & $\begin{array}{c}\text { Reduction } \\
(\%)\end{array}$ \\
\hline 1 & $m=1024, n=1$ & 1025 & 1025 & 1025 & 0.00 \\
2 & $m=9, n=2$ & 1023 & 1023 & 633 & 38.12 \\
3 & $m=6, n=3$ & 1093 & 1093 & 657 & 39.89 \\
4 & $m=5, n=4$ & 1365 & 1365 & 814 & 40.37 \\
5 & $m=4, n=6$ & 1555 & 1555 & 889 & 42.83 \\
\hline
\end{tabular}

Table 1 shows that the number of agents generated is reduced when $n>1$. When $n=1$, the number of agents generated is not reduced because the structure of the plan resembles a total order of tasks. The results show that as the value of $n$ increases the number of agents generated is significantly reduced.

\section{B. MCA Workload Test}

In this test, the MCA's workload is observed and studied. This test is conducted over a concern that MCA can become the bottleneck of the overall system performance. The metric used in this test is the time taken for a message to do a round trip. A message is sent from an external application to the MCA which in turn sends it back to the external application. These send/receive actions are continuously executed until the mission is completed. The hypothesis is, the message turnaround time increases as the MCA's workload gets higher.

To create various plan structures, we use three parameters: $m, n$ and $o$. Parameter $m$ is used to specify the depth of the TDG, parameter $n$ is used to specify the number of subtasks that the non-root tasks can have, and finally, parameter $o$ is used to specify the number of subtasks the root task can have. The test is conducted with two types of execution load: normal and heavy loads. The normal load represents a typical load of normal operations. In this type of operation, each agent will spend around two to five seconds executing some calculation logic. The heavy load represents the load of the very computationally intense operations. Under this kind of load the MCA normally becomes highly busy and occupied.

For each of the load types; the MCA executes the plans with and without the optimization routine. The turn around time unit is in milliseconds and it indicates the MCA's workload the shorter turn around time, the lighter MCA's workload is. The results of this experiment are presented in Table 2. The results show that on average message turn around time is smaller when the MCA performs the optimization routine compared to when it does not. This is because that even though the MCA needs to spend some time in performing the optimization, it actually spends less time in coordinating and managing the agents since they are fewer agents to coordinate (or communicate with).

TABLE 2

\begin{tabular}{ccccccc}
\multicolumn{7}{c}{ MCA WORKLOAD TEST RESULTS } \\
\hline \multirow{2}{*}{ Test No } & $\begin{array}{c}\text { Plan Structure } \\
\text { Parameters }\end{array}$ & $\begin{array}{c}\text { No Of } \\
\text { Tasks }\end{array}$ & $\begin{array}{c}\text { Normal Load (mSec) } \\
\text { Without } \\
\text { Optimization }\end{array}$ & $\begin{array}{c}\text { With } \\
\text { Optimization }\end{array}$ & $\begin{array}{c}\text { Without } \\
\text { Optimization }\end{array}$ & $\begin{array}{c}\text { With } \\
\text { Optimization }\end{array}$ \\
\hline 1 & $\mathrm{o}=5, \mathrm{n}=2, \mathrm{l}=3$ & 16 & 154 & 104 & 205 & 147 \\
2 & $\mathrm{o}=10, \mathrm{n}=2, \mathrm{l}=3$ & 31 & 175 & 103 & 211 & 149 \\
3 & $\mathrm{o}=25, \mathrm{n}=2, \mathrm{l}=3$ & 76 & 155 & 112 & 238 & 159 \\
4 & $\mathrm{o}=50, \mathrm{n}=2, \mathrm{l}=3$ & 151 & 158 & 113 & 244 & 151 \\
5 & $\mathrm{o}=50, \mathrm{n}=2, \mathrm{l}=4$ & 351 & 173 & 110 & 224 & 138 \\
6 & $\mathrm{o}=50, \mathrm{n}=2, \mathrm{l}=5$ & 751 & 165 & 103 & 233 & 143 \\
7 & $\mathrm{o}=50, \mathrm{n}=2, \mathrm{l}=6$ & 1551 & 159 & 111 & 241 & 153 \\
\hline
\end{tabular}

\section{RELATED WORK}

The concept of decomposing a goal into a set of tasks was initially addressed by HTN [7]. HTN is intuitive in that it resembles how human agents perform the practical planning. However, our study indicates that it does not meet the requirement of our project due to the following reasons: (i) a plan in HTN is a sequence of partially ordered tasks [8]. Due to this nature, there is some difficulty in using this representation as a plan for a group of agents (global plan). The difficulty arises when one needs to find the logical boundary from the task sequence to appropriately perform the task allocation to the agents, and (ii) since the final result of the HTN plan is a series of tasks, then it is difficult to provide run-time plan modification support such as adding and removing a group of tasks or sub-plans.

TÆMS [9] has certainly influenced our formal model in particular with respect to the notion of dependency between tasks. However, we found that TÆMS was: (i) too complex for our purposes in that even to design a simple plan one must consider all the quantitative distributions between tasks and resources beforehand, (ii) TÆMS does not provide missionbased plan but only represents plans for individual agents. In our case, the MCA in the community has overall control over the mission's accomplishment, (iii) TÆMS does not provide run-time plan modification, and finally (iv) since TÆMS is 
primarily focused on modeling the task structure, it does not provide any mechanism where agents can be automatically generated from the task structure.

\section{CONCLUSION AND FUTURE WORK}

The notions of mission-based MAS and on-demand agent generation have been presented in this paper. The formal models of the mission and TDG as well as mission execution and run-time plan modification have also been presented. The on-demand agent generation notion permits agents to be constructed at run-time just when it is needed while the concept of the mission provides an abstraction over the MAS and allows the MAS to be suspended, resumed and moved to different locations if necessary. The on-demand agent generation system can be offered as a pervasive service to software entities as well as to human users given that the front end interface is developed.

Developing a time and resource efficient strategy for allocating TDG tasks to agents is a key in realizing our mission-based concept of MAS. Our experimental results showed that the Bin-Packing strategy can reduce the number of agents generated without increasing the mission elapsed time, thereby cost-efficient. The reduced number of agents also means lighter overall workload to the MCA, despites of additional activities that must be carried out when the mission is run cost-efficiently. These additional activities are sorting, task clustering and Bin-Packing.

We also note that the agents we described can be interfaced with Web services. These agents can be assigned tasks which invoke Web services locally on the servers they visit. Hence, a mission can be considered a way to specify how a set of services can be integrated towards a specific purpose.

There is a need for high-level abstract view of what the user wants done (one could call this a service-based view) and integration techniques, when users are faced with a multitude of devices, Internet resources or services. The mission abstraction is one such candidate for defining such a view, and we have presented a precise description of this view. The subsequent challenge is then translating or processing the userspecified mission into actual computations whether on one device or multiple devices - the eHermes prototype presents one approach to processing missions. Analogous to compiling a program in a high level programming language into different kinds of platforms and hosts for execution, we process missions into agents which can execute the computations. In principle, such missions can be processed into agents that run on stationary hosts, mobile host, embedded computers, or even nanodevices. The agent is also a suitable abstraction in which to embed different kinds of functionality - as part of the same mission, different agents can be generated, one to invoke Web services, another to query databases, a third to query sensors, and a fourth to interact with other users - all such heterogeneity will be hidden from the user who sees the mission. For example, consider this mission entitled "celebrate passing of my exam" which is roughly stated as "check my exam results, and if I passed, order a bottle of champagne and deliver it to my house, when it arrives, ask the delivery person for the password, and if the right password is given, allow the person to leave the bottle on the floor mat near the door, inform me when the bottle touches the floor mat, and invite my friends too." Note, such a mission can be formalized into a TDG and agents generated must interact with different devices (from exam Web service, the grocery shop's Web service, the sensors embedded in my floor mat, to an event invitation service).

Future work for this project includes: (i) improvement in the Cost/Benefit Analysis so that it also takes vertical dependencies into consideration, (ii) allowing a plan to be divided into smaller sub-plans and create MCA clones for those sub-plans, and finally (iii) providing support and mechanism to re-use agents dynamically - hence, agents are not removed once they have completed the tasks but are given with a new set of tasks, and (iv) packaging the on-demand agent generation system as a pervasive service as well as developing a set of API for the service.

\section{ACKNOWLEDGMENT}

Support for this project from the Australian Research Council (ARC) Linkage Project scheme and Microsoft Research Asia Grant LP0211384 is thankfully acknowledged.

\section{REFERENCES}

[1] G. T. Jayaputera, A. Zaslavsky, and S. W. Loke, "An Approach to Dynamically Generated User-Specified MAS," LNCS, vol. 3950, pp 139-153, 2005

[2] A. E. Boardman, D. H. Greenberg, A. R. Vining, and D. L. Weimer, Cost-Benefit Analysis: Concepts and Practice, $2^{\text {nd }}$ ed: Prentice Hall, 2000.

[3] S. Martello and P. Toth, Knapsack Problems: Algorithms and Computer Implementations: John Wiley \& Sons, 1990.

[4] G. T. Jayaputera, S. W. Loke, and A. Zaslavsky, "Just in Time Mobile Agent Generation and Management," presented at $4^{\text {th }}$ International Joint Conference on Autonomous Agents and Multiagent Systems (AAMAS 05), Utrecht, Netherlands, 2005.

[5] T. Magedanz and C. Bäumer, "Grasshopper - A Universal Agent Platform Based on OMG MASIF and FIPA Standards," in http://www.cordis.lu/infowin/acts/analysys/products/thematic/agents/c h4/ch4.htm, 1999.

[6] B. Segall, D. Arnold, J. Boot, M. Henderson, and T. Phelps, "Content Based Routing with Elvin4," presented at Australian UNIX and Open Systems User Group Conference 2000 (AUUG 2000), Canberra, Australia, 2000.

[7] R. Callan, Artificial Intelligence: Palgrave Macmillan, 2003.

[8] K. Erol, J. Hendler, and D. Nau, "Semantics for Hierarchical TaskNetwork Planning," University of Maryland CS-TR-3239, UMIACSTR94-31, ISR-TR-95-9, 19941994.

[9] K. S. Decker, "TÆMS: A Framework for Environment Centered Analysis \& Design of Coordination Mechanisms," in Foundations of Distributed Artificial Intelligence, G. O'Hare and N. R. Jennings, Eds.: John Wiley \& Sons, 1996, pp. 429-447. 